\title{
Damage Identification by Using a Self-Synchronizing Multipoint Laser Doppler Vibrometer
}

\author{
Chong Yang, ${ }^{1,2,3}$ Yu Fu, ${ }^{2}$ Jianmin Yuan, ${ }^{2}$ Min Guo, ${ }^{2}$ Keyu Yan, ${ }^{2}$ Huan Liu, ${ }^{2}$ \\ Hong Miao, ${ }^{3}$ and Changchun $\mathrm{Zhu}^{1}$ \\ ${ }^{1}$ Institute of Systems Engineering, China Academy of Engineering Physics, Mianyang 621900, China \\ ${ }^{2}$ Temasek Laboratories and School of Mechanical \& Aerospace Engineering, Nanyang Technological University, \\ 50 Nanyang Drive, Singapore 637553 \\ ${ }^{3}$ Department of Modern Mechanics, University of Science and Technology of China, Hefei 230027, China
}

Correspondence should be addressed to Chong Yang; yang789@mail.ustc.edu.cn

Received 17 June 2014; Accepted 15 November 2014

Academic Editor: Hassan Haddadpour

Copyright (c) 2015 Chong Yang et al. This is an open access article distributed under the Creative Commons Attribution License, which permits unrestricted use, distribution, and reproduction in any medium, provided the original work is properly cited.

\begin{abstract}
The vibration-based damage identification method extracts the damage location and severity information from the change of modal properties, such as natural frequency and mode shape. Its performance and accuracy depends on the measurement precision. Laser Doppler vibrometer (LDV) provides a noncontact vibration measurement of high quality, but usually it can only do sampling on a single point. Scanning LDV is normally used to obtain the mode shape with a longer scanning time. In this paper, a damage detection technique is proposed using a self-synchronizing multipoint LDV. Multiple laser beams with various frequency shifts are projected on different points of the object, reflected and interfered with a common reference beam. The interference signal containing synchronized temporal vibration information of multiple spatial points is captured by a single photodetector and can be retrieved in a very short period. Experiments are conducted to measure the natural frequencies and mode shapes of pre- and postcrack cantilever beams. Mode shape curvature is calculated by numerical interpolation and windowed Fourier analysis. The results show that the artificial crack can be identified precisely from the change of natural frequencies and the difference of mode shape curvature squares.
\end{abstract}

\section{Introduction}

The structural damages, caused by aging, mechanical vibration, shock and chemical attack, and so forth, can debase the mechanical properties of structure and even yield catastrophic failures, and thus detecting the structural damage at earliest possible stages is significant in engineering related fields. Conventional nondestructive testing techniques, such as X-ray imaging, ultrasonic scans, and eddy current inspection, require the approximate flaw area to be known in advance and accessible for testing. Consequently, the vibration-based damage identification, as a global approach, has attracted the scientific and technical community over the past few decades [1-5]. The foundational principle is that the damage exerts effect on the physical properties of structure such as damping and stiffness and further changes the modal properties; thus, inversely, it can be diagnosed by analyzing the difference between modal properties before and after the defect occurrence.

Generally, there are two categories of currently used damage detection methods, frequency-based method and mode shape-based method. The former reaps the benefit of easy and precise natural frequency measurement; however, its practicability is limited by the following disadvantages: (1) the frequency-based method can be only applied to typical structures and damages which can be theoretically modeled via mathematical approximation; (2) damage induced frequency change may be very small even if the damage is considerable, and thus the method is easy to be affected by other factors such as the temperature variation; (3) different damages may cause identical frequency change especially when they occur on a symmetrical structure. In order to 
overcome these problems, mode shape-based approach is presented, since mode shape is globally distributed and more robust to environmental conditions.

In practice, mode shape derivative-based indicators, such as mode shape curvature (MSC) [6-8], strain mode shape, and modal strain energy (MSE) [9], are more commonly in use due to their higher sensitivities to damage than displacement mode shape [10]. MSC is the second-order spatial derivative of displacement mode shape. For an EulerBernoulli beam, the surface strain under bending is proportional to the curvature, so the strain mode shape and MSE can be regarded as special forms of MSC.

MSC can be computed from the measured displacement mode shape by finite difference approximation or other algorithms or determined by directly measuring the strain mode shape. However, either the displacement mode shape or the strain mode shape is much more complicated to be measured than the natural frequency. The electrical measuring method is most popular these days, such as commonly using accelerometers for displacement metrology or using strain gauges [11] or piezoelectric strain sensors [7, 12] for strain metrology. However, the electrical method is a contact measurement, in which the additional sensor mass restricts density of sensor placing and influences metrical precision. Besides, it is easily influenced by electromagnetic interference.

Numerous investigations have been conducted on damage diagnosis via optical measuring techniques. Optical fiber sensors, in particular the distributed fiber sensors which have a long strain sensing range along the entire fiber, have gained plenty of interest $[13,14]$. They are immune to electromagnetic interference but still need adhering to the target. The noncontact optical measuring techniques, such as digital image correlation (DIC) method [15], full-field optical interferometry $[16,17]$, and laser Doppler vibrometer (LDV), also have been paid more and more attention. DIC and fullfield interferometry employ the high-speed camera to record digital images. The camera provides a measurement with high resolution and sampling rate in spatial domain, but at present its temporal resolution and sampling rate are still low. Moreover, the measurement calls for a high stability to prevent environmental vibration and a lighting condition to capture the image.

Photodetector-based LDV can offer a much higher temporal sampling rate and demands less for stability and lighting contrasted with camera-based full-field interferometry. The dominating demerit is LDV which can only play a pointwise measurement as a single-pixel detector. For mode shape measurement, usually a scanning LDV (SLDV) [18] is adopted to measure the vibration on different spatial points. Ability of providing extremely high sampling density makes SLDV a popular metrical tool in damage identification [12, 19-22]. However, the scanning procedure is time consuming, and the accuracy relies on the stability and repeatability of the scanning device which utilizes mirrors to shift the measurement laser beam. The asynchronous sampling is more sensitive to noise. An instantaneous holistic rigid move of structure or metrical system can be eliminated in synchronous sampling, but it may engender a perturbation in asynchronous

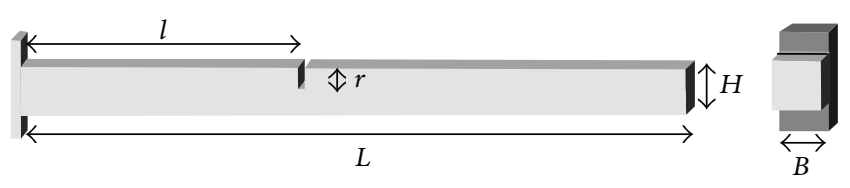

Figure 1: An Euler-Bernoulli cantilever beam model with a single crack.

sampling. Moreover, unlimited sampling points are unnecessary in respect that the quality of damage detection is not always improved with the increase of sampling, in practice [22].

In our previous research, a multipoint LDV was proposed to realize a twenty-point synchronized measurement in the form of a $5 \times 4$ beam array $[23,24]$. The beam array limits the metrical flexibility. To solve this problem, the laser beams are recoupled into optical fibers; hereby, for each channel, a fiber-based interferometer is formed to do measurement independently [25]. This multipoint LDV is potential in mode analysis. In this paper, a 14-point fiber-based LDV is applied to diagnose the crack in beam-like structure using a combination of frequency-based and mode shape-based methods. The damage location is determined by the change of MSC squares, and the severity is evaluated using a proposed frequency-based method. Numerical interpolation and windowed Fourier transform are presented to MSC computation, in order to prevent the noise magnification in differentiation. The results show that an accurate identification of both damage location and depth information can be achieved by using the proposed multipoint LDV system.

\section{Theoretical Analysis of Damage Identification}

2.1. Frequency-Based Damage Severity Analysis. The basic theory of vibration-based fault detection method is established on the characteristics of beam-like structures [26]. Figure 1 shows an Euler-Bernoulli cantilever beam model with a single notch crack. The length of the beam is $L$ and the crack of a depth $r$ is at the position of distance $l$ to the clamping end. Normally the ratio $e=l / L$ is used to represent the crack relative location and the relative depth $\alpha=r / H$ is used to represent the crack severity.

Mostly, the crack is assumed to be fully open during the vibration and equivalent to a rotational spring at the crack location, and the cracked beam is considered as two uniform beams connected by the spring [27-29]. The vibration equation of an Euler-Bernoulli beam is then valid for each segment of the beam separately, with the appropriate boundary condition. For such a beam-like structure, the relationship between natural frequency change ratio and the nondimensional spring stiffness $K$ is [29] as follows:

$$
\frac{\Delta f_{n}}{f_{u n}}=2 g_{n}(x) \frac{1}{K},
$$

where $\Delta f=f_{u}-f_{d}$ is the natural frequency change before and after the damage occurrence, subscript $n$ demotes the $n$th 
mode, and subscripts $u$ and $d$ imply the undamaged case and damaged case, respectively. The function $g_{n}(x)$ is

$$
g_{n}(x)=\frac{1}{4} \frac{\left.\left[\phi_{n}^{\prime \prime}(x)\right]^{2}\right|_{x=e}}{\int_{0}^{1}\left[\phi_{n}^{\prime \prime}(x)\right]^{2} d x}
$$

where $\phi_{n}$ is the $n$th mode shape, and for a cantilever beam, it can be expressed as

$$
\begin{aligned}
\phi_{n}(x)= & r_{n}\left[\sin \left(\beta_{n} x\right)-\sinh \left(\beta_{n} x\right)\right] \\
& +\cos \left(\beta_{n} x\right)-\cosh \left(\beta_{n} x\right),
\end{aligned}
$$

where

$$
\begin{aligned}
& r_{n}=\frac{\sin \left(\beta_{n}\right)-\sinh \left(\beta_{n}\right)}{\cos \left(\beta_{n}\right)+\cosh \left(\beta_{n}\right)}, \\
& \beta_{n}= \begin{cases}1.875 & n=1 \\
4.694 & n=2 \\
\left(n-\frac{1}{2}\right) \pi & n \geq 3 .\end{cases}
\end{aligned}
$$

The nondimensional spring stiffness $K=K_{r} L / E I$, where $K_{r}=E B H^{2} / 72 \pi \alpha^{2} f(\alpha)$ and $f(\alpha)=0.6384-1.035 \alpha+$ $3.7201 \alpha^{2}-5.1773 \alpha^{3}+7.553 \alpha^{4}-7.332 \alpha^{5}+2.4909 \alpha^{6}$. Letting these values into (1) and (2), the following can be obtained:

$$
\frac{\Delta f_{n}}{f_{u n}}=2 g_{n}(e) \frac{6 \pi H \alpha^{2} f(\alpha)}{L}=37.6991 \frac{g_{n}(e) H \alpha^{2} f(\alpha)}{L} \text {. }
$$

For the first mode being selected $(n=1), \beta_{1}=1.8751$, and hence the function $g_{n}(x)$ becomes

$$
g_{1}(e)=\frac{1}{4} \frac{\left[\phi_{1}^{\prime \prime}(e)\right]^{2}}{12.3623}=\frac{\left[\phi_{1}^{\prime \prime}(e)\right]^{2}}{49.4492} .
$$

Putting it in (5), we can get

$$
\frac{\Delta f_{1}}{f_{u 1}}=0.7624 \frac{\left[\phi_{1}^{\prime \prime}(e)\right]^{2} H \alpha^{2} f(\alpha)}{L}
$$

When the second mode shape is selected $(n=2), \beta_{2}=$ 4.6941, and thus we can get

$$
\begin{aligned}
\frac{\Delta f_{2}}{f_{u 2}} & =g_{2}(e) \frac{37.6991 H \alpha^{2} f(\alpha)}{L} \\
& =0.0194 \frac{\left[\phi_{2}^{\prime \prime}(e)\right]^{2} H \alpha^{2} f(\alpha)}{L} .
\end{aligned}
$$

The relationship between higher natural frequency, relative crack location, and relative crack depth can be derived from (1) and (2) according to the above deduction. If the natural frequency and crack location $e$ are known, the relative crack depth $\alpha$ can be calculated by (7) or (8).
2.2. Mode-Shape-Based Damage Localization. The mode shape variation happens with the defect-induced changes in dynamic characteristics of the beam. The damage alters the local stiffness, and thus subsequently the local mode shape changes. Mode shape derivatives are more commonly in use especially the MSC, the second-order spatial derivative of mode shape. Absolute difference of MSC squares (MSCS) [20] between intact and damaged structures can be used as the damage indicator:

$$
\operatorname{MSCS}=\left|\left(\phi_{u}^{\prime \prime}\right)^{2}-\left(\phi_{d}^{\prime \prime}\right)^{2}\right|
$$

The healthy structure's mode shape, used as the baseline, can be measured experimentally or shaped via numerical simulation. Notwithstanding in most real applications, the baseline is unavailable to be measured, and this paper is principally focused on metrical technique; thus, the baseline is acquired by experiment.

General algorithm to compute MSC is the central difference approximation:

$$
\phi^{\prime \prime}(k)=\frac{\phi(k+1)-2 \phi(k)+\phi(k-1)}{h^{2}},
$$

where $k$ implies the $k$ th sampling point and $h$ is the uniform distance between the sampling points. The algorithm is simple, but unfortunately it amplifies the measurement errors, especially when the spatial sampling points is sparsely placed. An improved way is computing the slope from curve fitting through the mode shape, but the error is still considerable.

In this paper, windowed Fourier ridges (WFR) algorithm [30] is introduced to extract MSC from the mode shape pretreated with a numerical interpolation. The interpolation inserts new data into the original sampling points to increase the number of data points. WFR transform the interpolated mode shape into its windowed Fourier amplitude spectrogram, searches out the maximum point at each specific spatial coordinate, and regards the corresponding frequency as the approximate local frequency. The algorithm has been proved to be a powerful tool to calculate the derivative of a signal when the window is selected appropriately [31]. For a signal in the form of $s(x)=A(x) \exp \{j \varphi(x)\}$, its windowed Fourier transform is

$$
\operatorname{WFR}(u, \xi)=\int_{-\infty}^{\infty} s(x) \exp \left\{-\frac{(x-u)^{2}}{2 \sigma^{2}}\right\} \exp \{-j \xi x\} d x
$$

where $\exp \left\{-(x-u)^{2} / 2 \sigma^{2}\right\}$ is the Gaussian window moving along the signal. Mathematically, the extraction of phase $\varphi(x)$ and phase derivative $\varphi^{\prime}(x)$ by WFR can be expressed as

$$
\begin{gathered}
\varphi(x)=\text { angle }\left.[\operatorname{WFT}(u, \xi)]\right|_{\xi=\varphi^{\prime}(u)}, \\
\varphi^{\prime}(u)=\left.\xi\right|_{\max \{\operatorname{abs}[\operatorname{WFT}(u, \xi)]\}} .
\end{gathered}
$$


Its principle of WFR is similar to that of wavelet ridges algorithm, but in respect that WFR take the exponential function as elemental function, as shown in (11), it is more suitable for a signal chiefly consisting of simple harmonic components. The mode shape is generally believed as such a signal in mathematics. Exemplarily, for an Euler beam-type structure, the analytical general expression of mode shape is a combination of trigonometric functions and hyperbolic functions, both of which can be converted into exponential expressions.

\section{Experimental Illustrations}

3.1. Fiber-Based Multipoint $L D V$. The principle of $L D V$ is based on the laser Doppler effect. For the same observer, when a laser beam with a frequency $f_{0}$ is projected onto a target with a velocity $V$, a Doppler frequency shift $f_{D}$ will happen to the reflected beam and $f_{D}$ is linearly related to the velocity:

$$
f_{D}(t)=2 \frac{f_{0}}{c} V(t) \cos \theta
$$

where $c$ is the speed of light and $\theta$ is the angle between illumination and observation direction. In LDV system, usually let $\theta=0$. If the Doppler frequency shift is already known, the velocity can be calculated by using (13). However, for the current luminous intensity detector, the laser frequency is too high to be directly measured, and the shift direction is impossible to be determined. One normally adopted problemsolving technique is heterodyne interferometry [32], which employs an acousto-optic modulator (AOM) to introduce an invariable frequency shift $f_{\mathrm{AOM}}$ into the object beam. Since $f_{D}+f_{\mathrm{AOM}}$ is extraordinarily small compared with the laser frequency, a beat effect will occur when the object beam interferes with the reference beam. In this case, a photodetector is able to record the intensity fluctuation. The beat frequency $f_{D}+f_{\mathrm{AOM}}$ can be acquired by a demodulation process. Since $f_{\mathrm{AOM}}$ is already known, if $f_{D}$ is between $-f_{\mathrm{AOM}}$ and $+f_{\mathrm{AOM}}$, the shift directional ambiguity can be solved and the velocity of object can be calculated by (13).

In our previous study, a spatially encoding technology was proposed to generate a beam array with various frequency shifts through a combination of AOMs at different diffraction types and realize a simultaneous vibration measurement on 20 points. Then a fiber-based configuration is adopted in optical design to couple the laser beams into different fibers and illuminate the various spatial points on the specimen by sensing heads. This configuration makes the system more flexible for various objects. The fiberbased LDV system in each channel can be treated as a separated subsystem. In this paper, a 14-point LDV is used to measure the modal characteristics of cantilever beams. Fourteen sensing heads will project 14 laser beams with frequency shifts from $30 \mathrm{MHz}$ to $290 \mathrm{MHz}$ with a frequency interval of $20 \mathrm{MHz}$ onto the object, and the reflected beams are interfered with a common reference beam. Interference intensity detected by the photodetector is

$$
\begin{array}{r}
I=I_{D C}+\sum_{i=1}^{14} \sqrt{I_{R} I_{O(i)}} \cos \left(2 \pi\left(f_{D(i)}+f_{\mathrm{AOM}(i)}\right) t+\Delta \psi_{(i)}\right) \\
+\sum_{p=1}^{13} \sum_{q>p}^{14} \sqrt{I_{\mathrm{O}(p)} I_{\mathrm{O}(q)}} \\
\times \cos \left(2 \pi \left[\left(f_{D(p)}-f_{D(q)}\right)\right.\right. \\
\left.\left.+\left(f_{\mathrm{AOM}(p)}-f_{\mathrm{AOM}(q)}\right)\right] t+\Delta \psi_{p q}\right),
\end{array}
$$

where $i=1,2,3, \ldots, 14, p$ and $q$ are integers, $\Delta \psi$ is the initial phase difference between the two interfering beams, $I_{O(i)}$ is the intensity of each object beam, and $I_{R}$ is the intensity of reference beam. The second term is the sum of interference signals between 14 object beams and the reference beam. The phase $f_{D(i)}+f_{\mathrm{AOM}(i)}$ includes the useful vibration information of 14 sampling points. However, while the object beams are interfering with the reference, the object beams interfere with each other to form the crosstalk as expressed in the third term. The intensity of the interference signals of the object beams and reference beam is determined by $\sqrt{I_{R} I_{O(i)}}$, while the intensity of the cross talk between any two object beams is determined by $\sqrt{I_{O(p)} I_{O(q)}}$. In that case, if the intensity of the reference is much higher than that of the object beams, the interference signals of the object beams and reference beam will be the dominating components in the spectrogram. By using a windowed Fourier ridge [30] or wavelet ridge algorithm to detect the signals with the highest energy at certain frequency range, the crosstalk can be bypassed and the vibration information on each measurement point can be demodulated. More details of the approach to eliminate the crosstalk are described in [33]. Since only one photodetector is used in this LDV system, synchronization problem is solved automatically. This system is applied to vibration measurement on a cantilever beam for crack inspection.

3.2. Experiments. An indefective beam and two cracked aluminum cantilever beams with the same dimensions and material properties are manufactured for experiments. The three-dimensional size of the beam is $270 \mathrm{~mm} \times 10 \mathrm{~mm} \times$ $10 \mathrm{~mm}$. An artificial single crack with a depth of $4 \mathrm{~mm}$ and a width of $0.2 \mathrm{~mm}$ is made on each cracked beam by the wire cutting technique. That means the relative crack depth $\alpha=$ 0.4. The cracks on the two beams are at position of $108 \mathrm{~mm}$ and $135 \mathrm{~mm}$ to the clamping end, respectively, and hence the crack location $e$ is, respectively, 0.4 and 0.5 . Fourteen equalspaced sample points are measured along the central line. The distances of sampling points to the clamping end are $70+(i-$ 1) $\times 10 \mathrm{~mm}$, where $i=1,2,3, \ldots, 14$. The experimental setup is shown in Figure 2.

In the first experiment, the natural frequencies of each cantilever beam are measured by the knocking method. A pendulum is used to apply a shock excitation to the beam at 


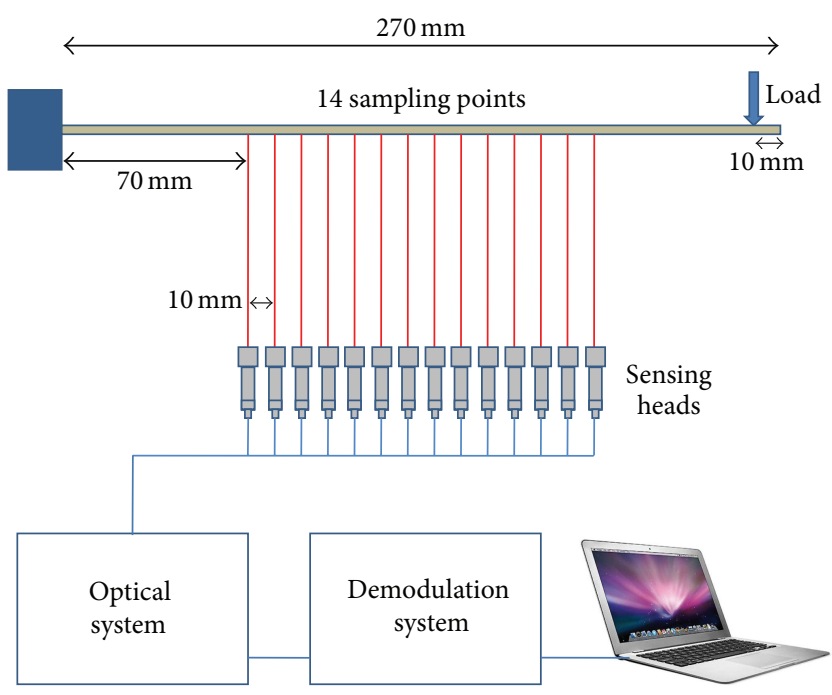

FIGURE 2: Experimental layout of the vibration measurment.

position of $10 \mathrm{~mm}$ from the free end. When the pendulum almost hits the beam, the digitizer is triggered and the temporal out-of-plane displacement variations on different sampling points are recorded. The natural frequencies can be extracted from spectrums of the displacement variations.

In the second experiment, the mode shape of each cantilever beam is measured by the proposed 14-point LDV system. The beam is subjected to an exciting force with sinusoidal configuration provided by an electromechanical shaker at position of $10 \mathrm{~mm}$ to the free end. Frequency of the exciting force is the corresponding second natural frequency of the beam determined in the above experiment, and thus the vibratory response is approximately the second mode shape.

The investigations on mode-shape-based damage identification are mostly devoted to the research of lower order modes, usually the first or second modes. It is so because the acquirement of lower modes is much more easier than that of higher ones, and there are less nodal points in lower mode shape, where the displacement is close to zero and thus the relative measurement error is high. However, the higher mode shape is more sensitive to the damage localization. Thus in the experiment, we select the second mode shape which is relatively a higher mode shape than the first mode shape.

\section{Results and Discussion}

Figure 3(a) shows the out-of-plane displacement of one sampling point in the first $0.08 \mathrm{~s}$ when the pendulum hits on the intact beam. From its spectrum, several resonances denoting structural natural frequencies can be observed. In order to increase the signal-to-noise ratio (SNR) of measurement, an average operation of multiple spectrums is conducted since the proposed LDV can provide a measurement on multiple points in a single test. Figure 3(b) shows the averaged spectrum, where the $y$-coordinate represents natural logarithm of the spectrum coefficient. The first and second natural
TABLE 1: Measured natural frequencies of the three beams.

\begin{tabular}{lcc}
\hline & $f_{1}(\mathrm{~Hz})$ & $f_{2}(\mathrm{~Hz})$ \\
\hline Intact beam & 105.6 & 663.6 \\
Cracked beam $(e=0.4)$ & 103.3 & 637.7 \\
Cracked beam $(e=0.5)$ & 104.1 & 633.2 \\
\hline
\end{tabular}

TABLE 2: Relative crack depth calculated by (7) and (8).

\begin{tabular}{lcc}
\hline & $\begin{array}{c}\text { Cracked beam } \\
(e=0.4)\end{array}$ & $\begin{array}{c}\text { Cracked beam } \\
(e=0.5)\end{array}$ \\
\hline$\alpha$ (actual) & 0.4 & 0.4 \\
First frequency change ratio & 0.0218 & 0.039 \\
$\alpha$ calculated by (7) & 0.353 & 0.367 \\
Relative error & $11.75 \%$ & $8.25 \%$ \\
Second frequency change ratio & 0.0142 & 0.0458 \\
$\alpha$ calculated by (8) & 0.382 & 0.333 \\
Relative error & $4.5 \%$ & $16.75 \%$ \\
\hline
\end{tabular}

frequencies of the intact beam are $105.5 \mathrm{~Hz}$ and $663.4 \mathrm{~Hz}$, respectively. The same data processing is conducted to the cracked beams and the average spectra of the corresponding beams with $e=0.4$ and $e=0.5$ are shown in Figures 3(c) and $3(d)$, respectively. The first and second frequencies of the three beams are showed in Table 1 .

In the second experiment, vibrations on 14 points of the beams are obtained. Figure 4 plots out-of-plane displacement variations of the first two sampling points on the indefective beam in the first $0.04 \mathrm{~s}$. Figure 5(a) shows the normalized second mode shape of undamaged beam, where the $x$ coordinate represents the relative position $e$. The value of each point is an averaged displacement in the same time duration. Figure 5(b) shows the interpolated mode shape by cubic spline interpolation, and Figure 5(c) is the MSC extracted by WFR algorithm. The same data processing is conducted to the cracked beams. The normalized 14point mode shapes, the interpolated versions, and the modal curvatures of cracked beams are shown in Figures 6(a), 6(b), and 6(c), respectively. Figure 6(d) shows the absolute difference of MSC squares of intact and defective beams. The maximum value of the difference can indicate the location of artificial crack precisely.

Based on the crack locations and natural frequencies information, the relative crack depth $\alpha$ can be calculated by (7) and (8), respectively, and is then shown in Table 2. The error is less than $20 \%$, which is acceptable in engineering applications. The error mainly comes from the difference between actual beam structure and theoretical model which relies on the theory of Euler-Bernoulli beam and rotational spring approximation. The three beams used for experiments may be slightly different in their actual dimensional sizes, material constants, and other variables. Furthermore, the clamping end of cantilever beam may not provide an exactly strict boundary condition as the theoretical description. For a complex structure in real application, the analytic solution is impossible to be deduced, and thus it is complicated and difficult to evaluate the damage severity quantitatively. 


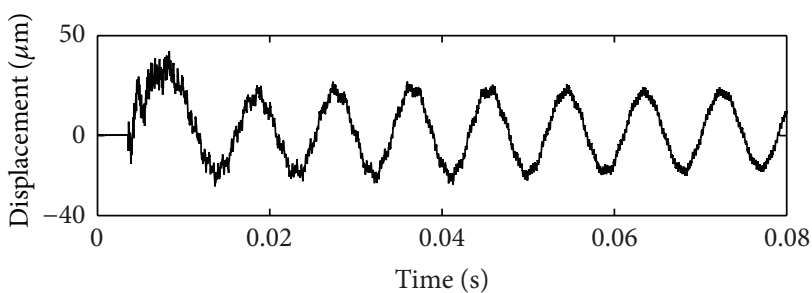

(a)

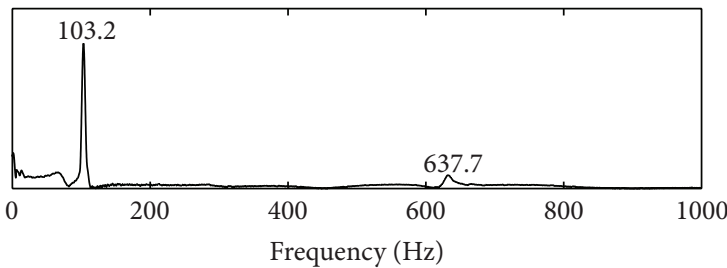

(c)

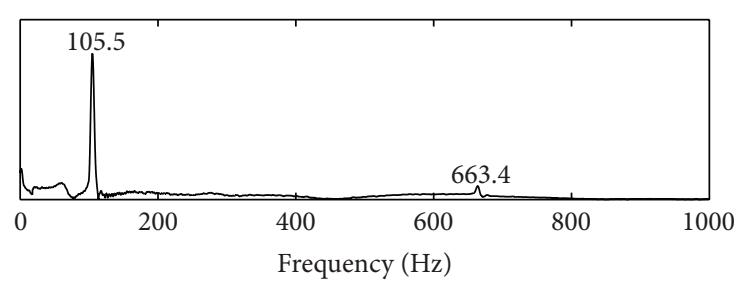

(b)

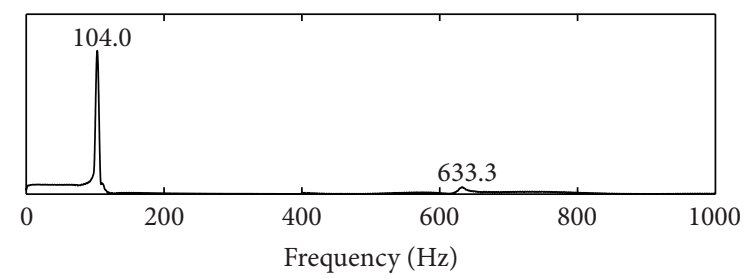

(d)

Figure 3: (a) Out-of-plane displacement distribution of one sampling point on the intact beam in the first $0.08 \mathrm{~s}$ after trigger; spectrum of the displacement distribution of (b) intact beam; (c) cracked beams with $e=0.4$; (d) cracked beams with $e=0.5$.

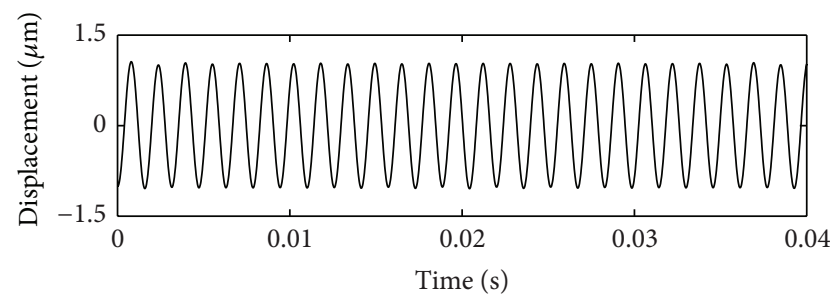

(a)

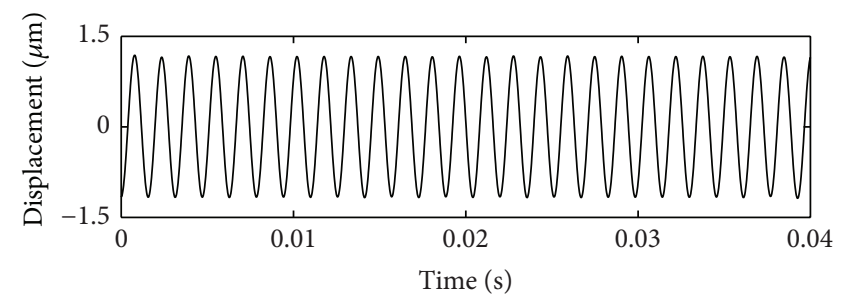

(b)

FIGURE 4: Out-of-plane displacement variation of the first two measurement points on the intact beam in first $0.04 \mathrm{~s}$.

An approximate estimation can be done according to the reduction of natural frequency, because the natural frequency decreases with the increase of damage severity.

Proper interpolation and differentiation algorithms are helpful to improve the precision of MSC calculation. The interpolation increases the number of spatial points of the signal and thus enhances the performance of windowed Fourier transform. Figures 5(c) and 6(c) show that WFR is capable of extracting the second derivative with a high precision. It can be observed that the crack position does not superimpose with the sampling points in the experiment, which demonstrates the combined use of interpolation and WFR algorithms promote the crack localization. However, primarily, the localization depends on the interval between sampling points, and, according to the discussion centered on the spatial sampling rate by Sazonov and Klinkhachorn [22], the number of measurement points provided by the proposed LDV is still less than the theoretical optimal value. Since the approach to remove crosstalk has been established, the number of measurement points is only restricted by the range of frequency and velocity of vibration to be measured. Considering that almost entirely the low-frequency vibration in a small scale is adopted in defect diagnoses, the number of measurement laser beams can be developed from current fourteen to forty and more. In that case, the inspection performance can be enhanced significantly. Besides, gradually zooming out the searching range is a good solution since the fiber-based system can adjust the spatial interval of measurement points conveniently.

\section{Concluding Remarks}

In this paper, a self-synchronizing multipoint LDV is proposed for vibration-based damage detection. Vibration information of 14 spatial points on the structure can be measured simultaneously. The location and severity of structural damage can be extracted from the changes of natural frequencies and MSC squares. Numerical interpolation and windowed Fourier transform are adopted to calculate the MSC from the measured mode shape and localize the flaw precisely. In the MSC-based damage detection method, a measurement of mode shape with a high quality is required. As the proposed fiber-based multipoint LDV can do vibration measurement simultaneously on multiple points, it avoids the influence of scanning mechanism and asynchronous sampling on mode shape measurement. In addition, it tremendously reduces the measurement time. Hence, it may gain more applications in damage diagnosis field due to its high flexibility and accuracy. No matter what damage indicator or signal processing algorithm is selected, a precise measurement of modal properties 


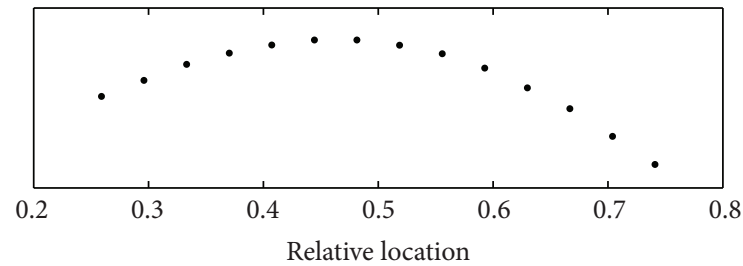

(a)

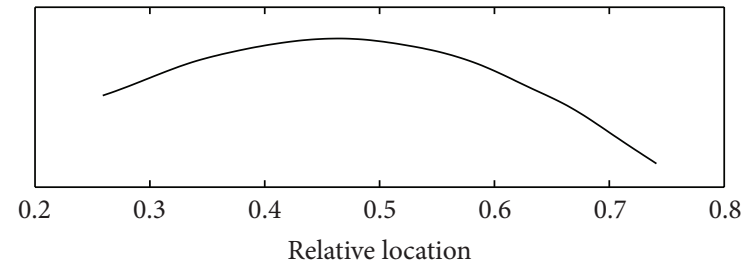

(b)

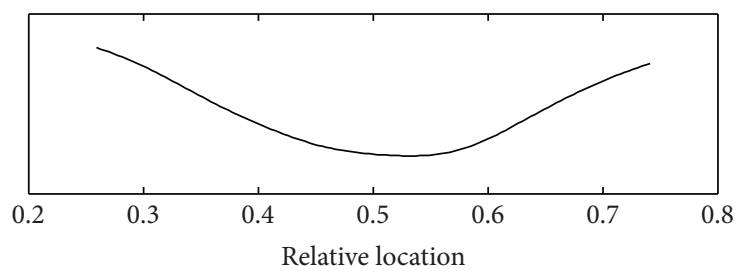

(c)

FIGURE 5: (a) 14-point mode shape of the intact beam; (b) interpolated mode shape of the intact beam; (c) MSC of the intact beam extracted by WFR method.
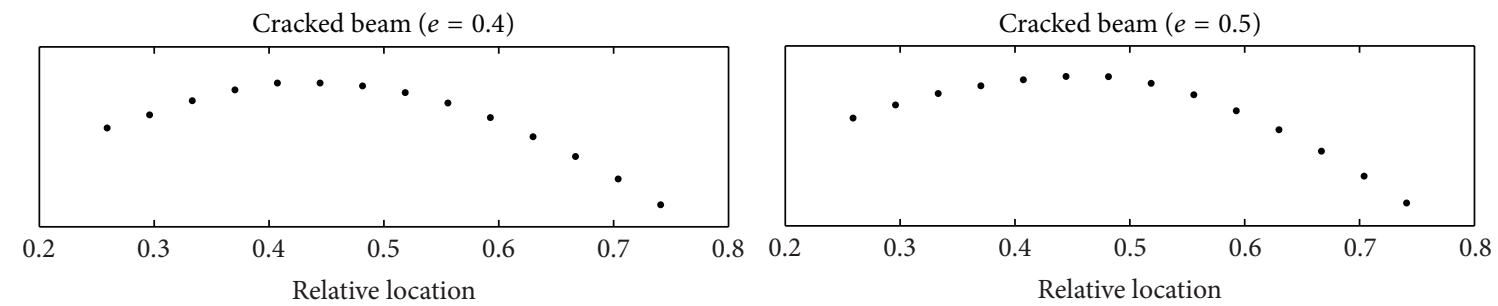

(a)
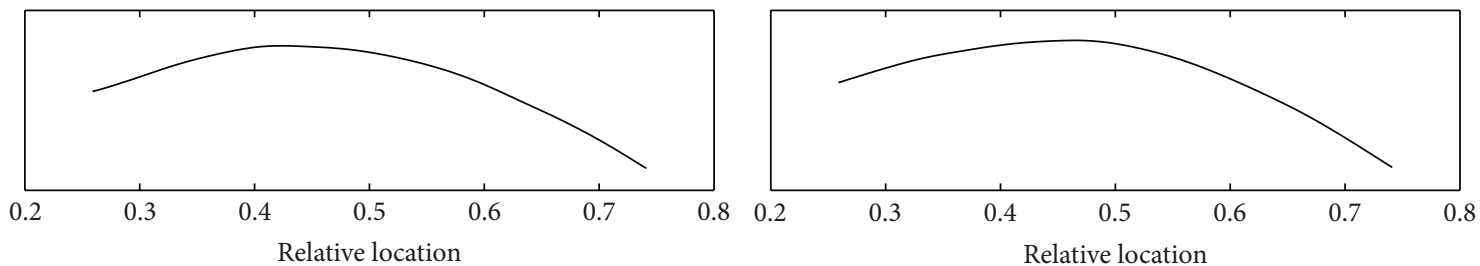

(b)
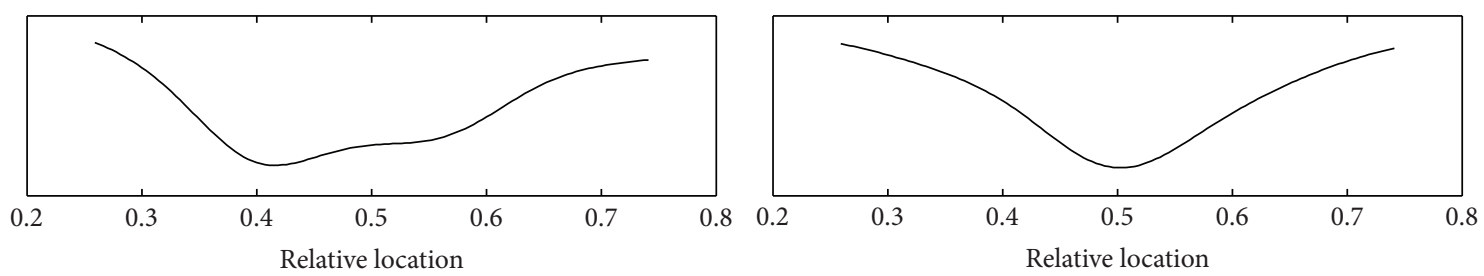

(c)
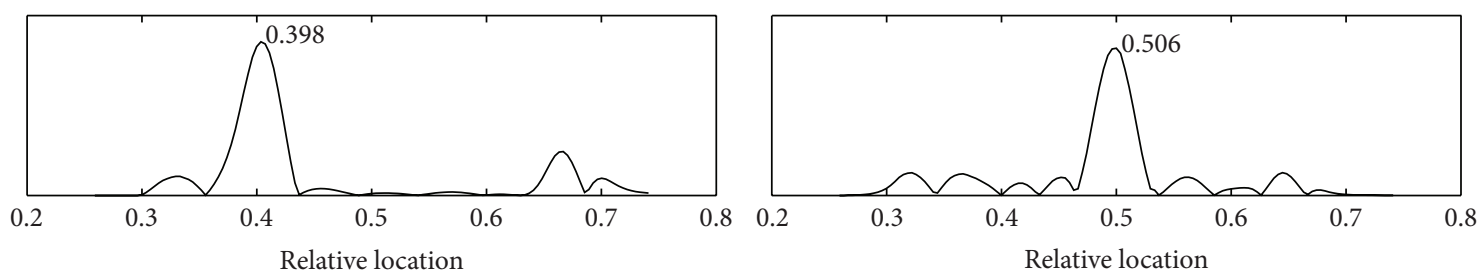

(d)

FIGURE 6: (a) 14-point mode shapes of the cracked beams; (b) interpolated mode shapes; (c) MSCs extracted by WFR method; (d) absolute difference of MSC squares of the intact and cracked beams. 
of the defective structure is significant to implement and accomplish the identification.

\section{Conflict of Interests}

The authors declare that there is no conflict of interests regarding the publication of this paper.

\section{Acknowledgments}

This work is mainly supported by Research Project TRF10MUPLAD, DRTech, and MINDEF, Singapore. Chong Yang and Hong Miao also acknowledge the financial supports provided by the National Science Foundation of China (NSFC) under Contracts nos. 11072235 and 10772171 and the Scientific Special Fund under Contract no. 2013KJZ10 funded by the Institute of Systems Engineering, CAEP.

\section{References}

[1] S. W. Doebling, C. Farrar, M. B. Prime, and W. S. Danial, "Damage identification and health monitoring of structural and mechanical systems from changes in their vibration characteristics: a literature review," Los Alamos National Laboratory Report LA-13070-MS, 1996.

[2] H. Sohn, C. R. Farrar, F. M. Hemez, D. D. Shunk, W. S. Daniel, and B. R. Nadler, "A review of structural health monitoring literature," Los Alamos National Laboratory Report LA-13976MS, 2003.

[3] J.-T. Kim, Y.-S. Ryu, H.-M. Cho, and N. Stubbs, "Damage identification in beam-type structures: frequency-based method vs mode-shape-based method," Engineering Structures, vol. 25, no. 1, pp. 57-67, 2003.

[4] P. J. S. Cruz and R. Salgado, "Performance of vibration-based damage detection methods in bridges," Computer-Aided Civil and Infrastructure Engineering, vol. 24, no. 1, pp. 62-79, 2009.

[5] W. Fan and P. Qiao, "Vibration-based damage identification methods: a review and comparative study," Structural Health Monitoring, vol. 10, no. 1, pp. 83-111, 2011.

[6] A. K. Pandey, M. Biswas, and M. M. Samman, "Damage detection from changes in curvature mode shapes," Journal of Sound and Vibration, vol. 145, no. 2, pp. 321-332, 1991.

[7] P. Qiao, K. Lu, W. Lestari, and J. Wang, "Curvature mode shape-based damage detection in composite laminated plates," Composite Structures, vol. 80, no. 3, pp. 409-428, 2007.

[8] M. Dilena and A. Morassi, "Dynamic testing of a damaged bridge," Mechanical Systems and Signal Processing, vol. 25, no. 5, pp. 1485-1507, 2011.

[9] P. J. Cornwell, S. W. Doebling, and C. R. Farrar, "Application of the strain energy damage detection method to plate-like structures," Journal of Sound and Vibration, vol. 128, pp. 521-529, 1999.

[10] H. Luo and S. Hanagud, "An integral equation for changes in the structural dynamics characteristics of damaged structures," International Journal of Solids and Structures, vol. 34, no. 35-36, pp. 4557-4579, 1997.

[11] Z. G. Zhou, L. D. Wegner, and B. F. Sparling, "Vibration-based detection of small-scale damage on a bridge deck," Journal of Structural Engineering-ASCE, vol. 133, no. 9, pp. 1257-1267, 2007.
[12] P. Qiao, W. Lestari, M. G. Shah, and J. Wang, "Dynamics-based damage detection of composite laminated beams using contact and noncontact measurement systems," Journal of Composite Materials, vol. 41, no. 10, pp. 1217-1252, 2007.

[13] M. Maheshwari, A. K. Asundi, and S. C. Tjin, "Effect of the location and size of a single crack on first fundamental frequency of a cantilever beam using fiber optic polarimetric sensors and characterisation of FBG sensors," in 4th International Conference on Smart Materials and Nanotechnology in Engineering, vol. 8793 of Proceedings of SPIE, Gold Coast, Australia, July 2013.

[14] A. P. Adewuyi and Z. S. Wu, "Modal macro-strain flexibility methods for damage localization in flexural structures using long-gage FBG sensors," Structural Control and Health Monitoring, vol. 18, no. 3, pp. 341-360, 2011.

[15] M. N. Helfrick, P. Pingle, C. Niezrecki, and P. Avitabile, "Using full-field vibration measurement techniques for damage detection," in Proceedings of the 27th Conference and Exposition on Structural Dynamics (IMAC '09), Orlando, Fla, USA, February 2009.

[16] H. Lopes, J. Ribeiro, and A. J. V. dos Santos, "Interferometric techniques in structural damage identification," Shock and Vibration, vol. 19, no. 5, pp. 835-844, 2012.

[17] A. M. Amaro, P. N. B. Reis, M. F. S. F. de Moura, and J. B. Santos, "Damage detection on laminated composite materials using several NDT techniques," Insight: Non-Destructive Testing and Condition Monitoring, vol. 54, no. 1, pp. 14-20, 2012.

[18] J. La, J. Choi, S. Wang, K. Kim, and K. Park, "Continuous scanning laser Doppler vibrometer for mode shape analysis," Optical Engineering, vol. 42, no. 3, pp. 730-737, 2003.

[19] V. K. Sharma, S. Hanagud, and M. Ruzzene, "Damage index estimation in beams and plates using laser vibrometry," AIAA Journal, vol. 44, no. 4, pp. 919-923, 2006.

[20] S. Rucevskis and M. Wesolowski, "Identification of damage in a beam structure by using mode shape curvature squares," Shock and Vibration, vol. 17, no. 4-5, pp. 601-610, 2010.

[21] M. Rucka, "Damage detection in beams using wavelet transform on higher vibration modes," Journal of Theoretical and Applied Mechanics, vol. 49, no. 2, pp. 399-417, 2011.

[22] E. Sazonov and P. Klinkhachorn, "Optimal spatial sampling interval for damage detection by curvature or strain energy mode shapes," Journal of Sound and Vibration, vol. 285, no. 4-5, pp. 783-801, 2005.

[23] Y. Fu, M. Guo, and P. B. Phua, "Spatially encoded multibeam laser Doppler vibrometry using a single photodetector," Optics Letters, vol. 35, no. 9, pp. 1356-1358, 2010.

[24] Y. Fu, M. Guo, and P. B. Phua, "Multipoint laser Doppler vibrometry with single detector: principles, implementations, and signal analyses," Applied Optics, vol. 50, no. 10, pp. 12801288, 2011.

[25] C. Yang, M. Guo, H. Liu et al., "A multi-point laser Doppler vibrometer with fiber-based configuration," Review of Scientific Instruments, vol. 84, no. 12, Article ID 121702, 2013.

[26] C. A. Papadopoulos and A. D. Dimarogonas, "Coupled longitudinal and bending vibrations of a rotating shaft with an open crack," Journal of Sound and Vibration, vol. 117, no. 1, pp. 81-93, 1987.

[27] P. F. Rizos, N. Aspragathos, and A. D. Dimarogonas, "Identification of crack location and magnitude in a cantilever beam from the vibration modes," Journal of Sound and Vibration, vol. 138, no. 3, pp. 381-388, 1990. 
Shock and Vibration

9

[28] B. P. Nandwana and S. K. Marti, "Detection of the location and size of a crack in stepped cantilever beams based on measurements of natural frequencies," Journal of Sound and Vibration, vol. 203, no. 3, pp. 435-446, 1997.

[29] F. B. Sayyad and B. Kumar, "Identification of crack location and crack size in a simply supported beam by measurement of natural frequencies," Journal of Vibration and Control, vol. 18, no. 2, pp. 183-190, 2012.

[30] Q. Kemao, “Two-dimensional windowed Fourier transform for fringe pattern analysis: principles, applications and implemenrations," Optics and Lasers in Engineering, vol. 45, no. 2, pp. 304317, 2007.

[31] C. Yang, Q. Lu, J. Zhao, and H. Mao, "Window size selection in windowed Fourier transform for phase retrieval," Optics and Lasers in Engineering, vol. 48, no. 11, pp. 1096-1103, 2010.

[32] G. Cloud, "Optical methods in experimental mechanics: part 17: laser doppler interferometry," Experimental Techniques, vol. 29, no. 3, pp. 27-30, 2005.

[33] Y. Pu, M. Goo, and P. B. Phua, "Cross-talk prevention in optical dynamic measurement," Optics and Lasers in Engineering, vol. 50, no. 4, pp. 547-555, 2012. 

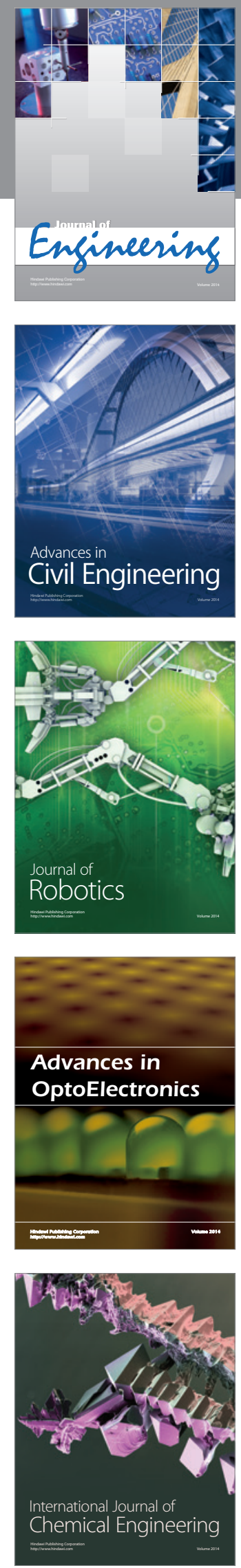

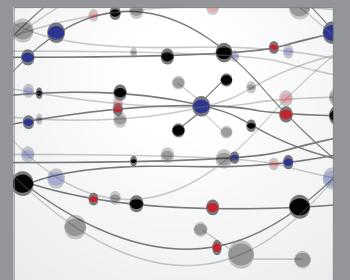

The Scientific World Journal
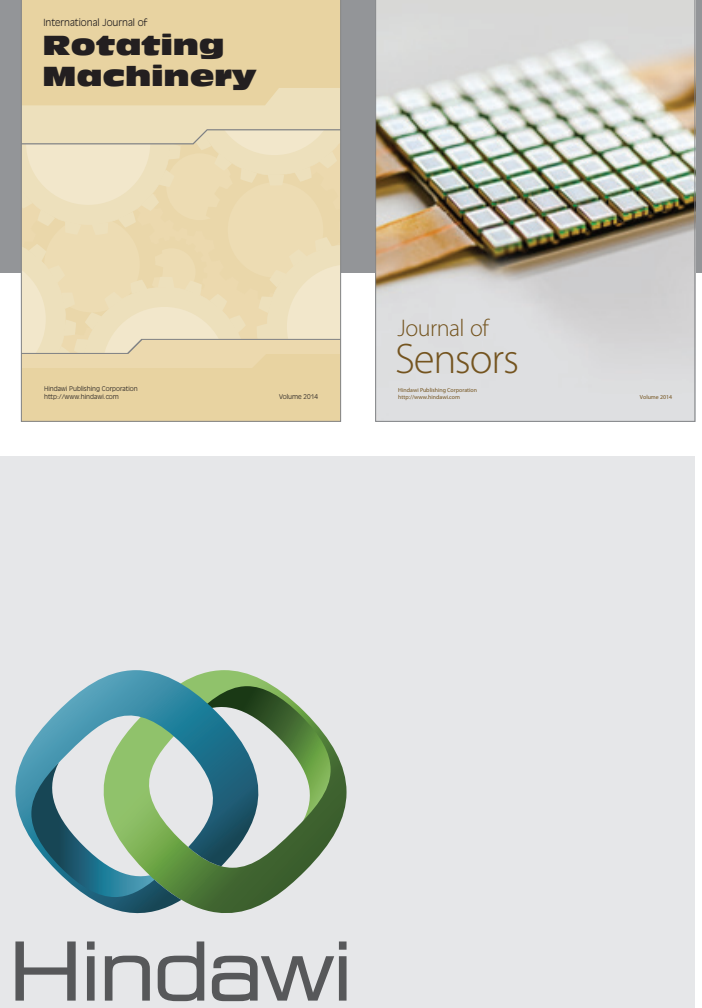

Submit your manuscripts at http://www.hindawi.com
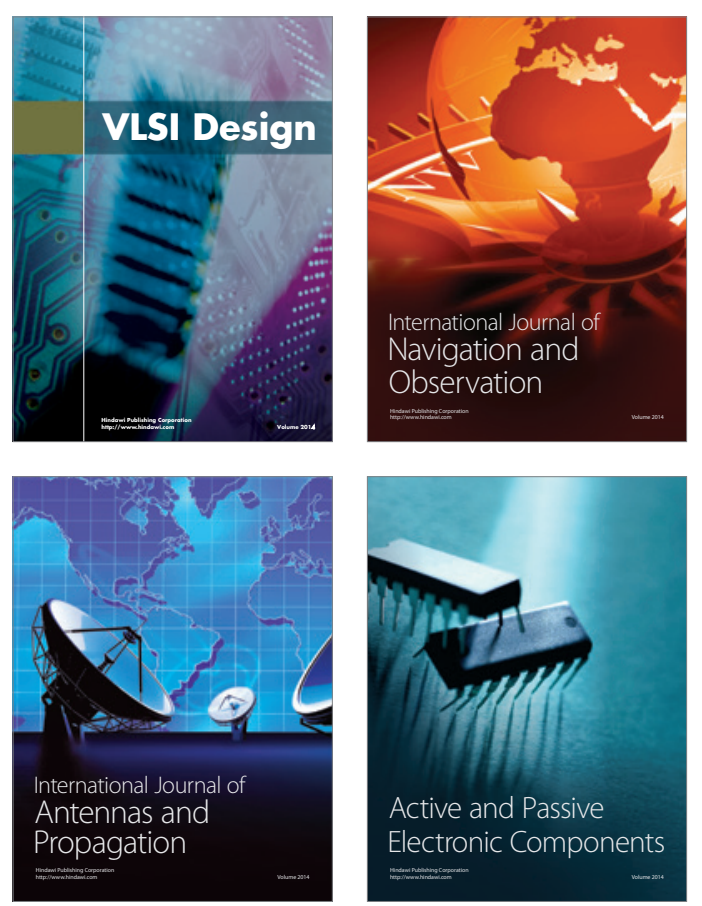
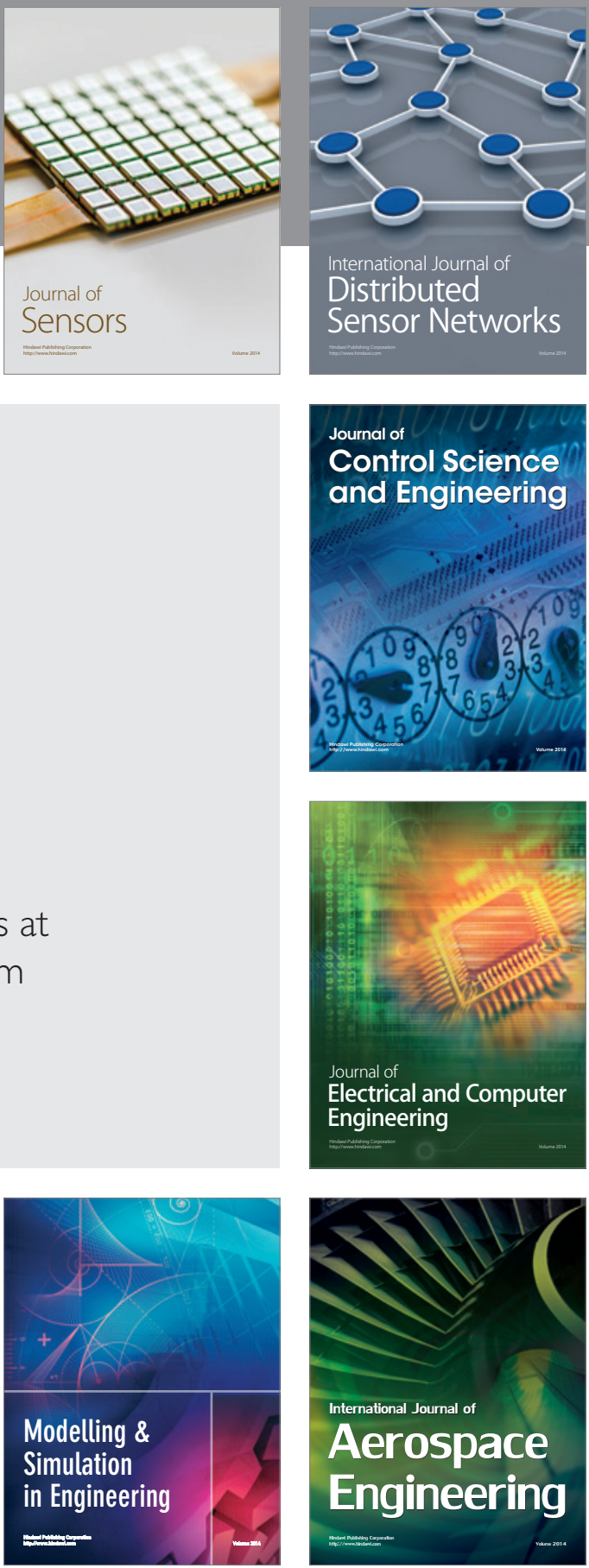

Journal of

Control Science

and Engineering
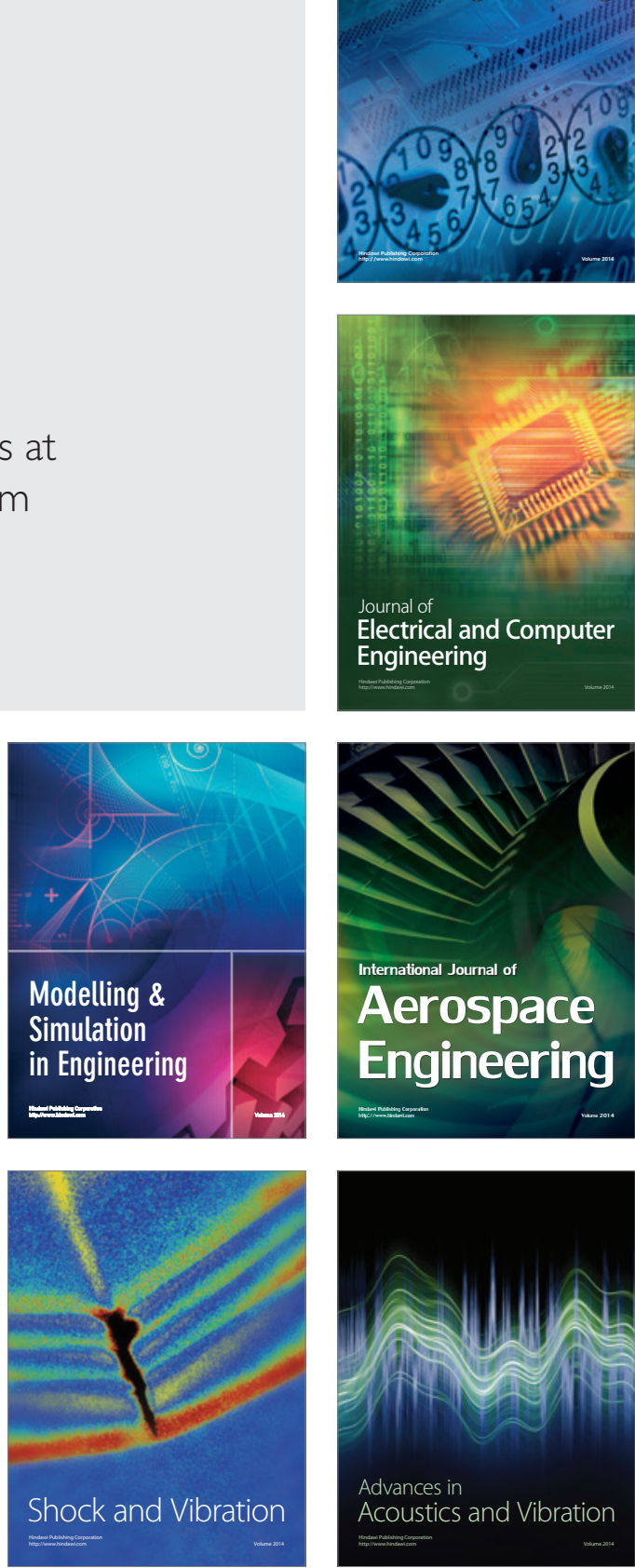